\title{
The Relationship between Decorin and VEGF in Endometriosis
}

\author{
Gultekin Adanas Aydin ${ }^{1}$, Habibe Ayvaci ${ }^{2}$ Nermin Koc ${ }^{3}$, Nazan Tarhan² and Oya Demirci ${ }^{2}$ \\ ${ }^{1}$ Department of Obstetrics and Gynecology, Bursa City Hospital, Bursa, Turkey \\ ${ }^{2}$ Department of Obstetrics and Gynecology, Zeynep Kamil Women's and Children's Disease Training and Research Hospital, Istanbul, \\ Turkey \\ ${ }^{3}$ Department of Pathology, Haydarpaşa Numune Training and Research Hospital, Istanbul, Turkey
}

\begin{abstract}
Objective: To evaluate immunohistochemical (IHC) staining of decorin and vascular endothelial growth factor (VEGF) of ovarian and endometrial tissues in patients with and without endometriosis.

Study Design: Descriptive study.

Place and Duration of Study: Department of Obstetrics and Gynecology, ZeynepKamil Training and Research Hospital, Istanbul, Turkey, between Istanbul, Turkeyjanuary 2018 and June 2019.

Methodology: Thirty patients, who underwent total abdominal hysterectomy (TAH) + bilateral salpingo-oophorectomy (BSO)/unilateral salpingo-oophorectomy (USO) and were in the proliferative phase of menstrual cycle,were included. The study population consisted of 20 patients (patient group) with an endometriomaand the control group consisted of 10 patients who were operated for benign gynecological pathologies. The ovarian and endometrial tissue specimens were collected from the archives. IHC staining was performed using decorin and VEGF.

Results: Decorin analysis showed a significantly higher intensity of staining in both endometrial and ovarian tissues in control group than patient group. Patients with endometriosis had a lower intensity of staining of decorin and a higher intensity of staining of VEGF compared to control group. There was a negative, statistically significant concordance between VEGF and decorin staining of endometrial tissues of both groups (concordance rate $-0.560, p=0.001$ ). Therewas a negative, statistically significant concordance between VEGF and decorin staining of ovarian tissues of both groups (concordance rate $-0.564, p<0.001$ ).

Conclusion: Angiogenesis plays a critical role in endometriosisand interaction between decorin and VEGF, which suggests that decorin may be a promising molecule for the treatment of endometriosis.
\end{abstract}

Key Words: Decorin, Endometriosis, Immunohistochemical staining, Vascular endothelial Growth factor.

How to cite this article: Aydin GA, Ayvaci H, Koc N, Tarhan N, Demirci O. The Relationship between Decorin and VEGF in Endometriosis. J Coll Physicians Surg Pak 2021; 31(11):1285-1290.

\section{INTRODUCTION}

Endometriosis is a benign condition characterised by the presence of endometrial tissue outside the uterine cavity, particularly on the ovaries. It affects $6 \%$ to $10 \%$ of all women of reproductive age. It is the most common cause of pelvic pain and is associated with infertility. ${ }^{1}$

Endometriosis is an estrogen-dependent, chronic inflammatory syndrome with a polygenic and multifactorial etiology. ${ }^{2}$ The retrograde menstruation theory is the most widely accepted theory in explaining etiology of endometriosis.

Correspondence to: Dr. Gultekin Adanas Aydin, Department of Obstetrics and Gynecology, Bursa City Hospital, Bursa, Turkey

E-mail: gadanas@gmail.com

Received: June 21, 2021; Revised: September 16, 2021;

Accepted: September 22, 2021

DOI: https://doi.org/10.29271/jcpsp.2021.11.1285
This theory proposes that endometriosis results from the retrograde flow of endometrial cells via the fallopian tubes into the pelvic cavity during menstruation. ${ }^{3}$ Although it is frequently seen in women, endometriosis occurs in only $10 \%$ of them. ${ }^{4}$ Endometriotictissues contain an abundantnumber of epigenetically abnormal stromal cells and few epithelial cells. ${ }^{4}$ Once the epigenetically abnormal cells reach the peritoneal cavity, they express the pro-survival genes such as steroidogenic factor-1 (SF-1) or estrogen receptor-beta (ER- $\beta$ ). ${ }^{5}$ In vitro studies have demonstrated that progenitor/stem cells in the basalis layer of endometrium, which play a key role in the regeneration of fullthickness mucosa every month with the effect of estrogen, do not convert into stromal cells in patients with endometriosis. ${ }^{6}$ In addition, inadequate differentiation of stromal cells is critical for resistance to progesterone receptors. ${ }^{7}$

According to the retrograde menstruation theory, angiogenesis plays a pivotal role in the growth and adherence of sloughed endometrial cells to the peritoneum. ${ }^{8}$ The vascular endothelial growth factor (VEGF) is a heparin-binding protein with angio- 
genic properties and activates endothelial cells, indicating a possible role in the pathogenesis and maintenance of endometriosis. $^{9}$

Besides soluble molecules such as VEGF and transforming growth factor-beta (TGF- $\beta$ ), extracellular matrix (ECM) macromolecules play a role in the angiogenesis. ${ }^{10}$ Decorin is a member of small leucine-rich proteoglycans and contains a core glycoprotein and chondroitin or dermatan sulfate glycosaminoglycan side chain. ${ }^{11}$ It is involved in the collagen fibrillogenesisand maintenance of tissue integrity, functioning as a reservoir for TGF$\beta .{ }^{11}$ Decorin is an antagonistic ligand for many tyrosine kinases including epidermal growth factor receptor (EGFR), Met, insulin-like growth factor-1 (IGFR-1), and VEGFR-2. ${ }^{12}$ It also induces autophagy in micro and macrovascular endothelial cells through VEGF-2 and inhibits angiogenesis. ${ }^{13}$

Previous studies have shown that decorin evokes anti-angiogenic and pro-autophagic effects by interacting with the VEGF; and angiogenesis plays a role in the pathogenesis of endometriosis similar to many inflammatory and ischemic diseases. 8

In the light of these data, the objective of the present studywas to analysethe differences in the immunohistochemical (IHC) staining of decorin and VEGF in patients with and without endometriosis were investigated and the possible correlation between the decorin and VEGF staining.

\section{METHODOLOGY}

This single-centre, descriptive study was conducted retrospectivelyat Obstetrics and Gynecology Clinic of ZeynepKamil Training and Research Hospital between January 2018 and June 2019. A total of 30 patients, who underwent total abdominal hysterectomy $(\mathrm{TAH})+$ bilateral salpingo-oophorectomy (BSO)/unilateral salpingo-oophorectomy (USO) and who were in the proliferative phase of the menstrual cycle, were included. Patients having malignant gynecological pathologies, receiving estrogen and/or progesterone within the past three months, receiving gonadotropin-releasing hormone analogues, or having a previous endometrioma surgery were excluded. The study population consisted of 20 patients (patient group) with an endometrioma of whom 11 had an accompanying uterine myoma and one had an endometrial polyp. The control group consisted of 10 patients who were operated for benign gynecological pathologies such as uterine myomas $(n=7)$, endometrial polyps $(n=1)$, and prolapse $(n=2)$, except for adnexal masses. Data including demographic and clinical characteristics of the patients were recorded using the hospital database. The ovarian and endometrial tissue specimens were collected from the archive; and IHC staining was performed using decorin and VEGF. A written informed consent was obtained from each patient. The study protocol was approved by the local Ethics Committee (6.3.2019 32). The study was conducted in accordance with the principles of the Declaration of Helsinki.

Hematoxylin and eosin ( $H \& E$ ) staining was performed for the histopathological examination using the formalin-fixed paraffin embedded tissue specimens. Endometriosis was diagnosed according to the presence of two or more following criteria suggested by the American College of Obstetricians and Gynecologists i.e. endometrial epithelium, endometrial glands, endometrial stroma, or hemosiderin laden macrophages. ${ }^{15}$ Thepatients with a uterine endometrial gland and stroma in the ovary were included.

For IHC staining, 3-micron thick sections were collected on positively charged glass slides and baked at $60^{\circ} \mathrm{C}$ for an hour in the oven. The slides were deparaffinised and rehydrated in distilled water. Then, the slides were immersed in the antigen retrieval buffer using a VEGF antibody (EDTA buffer, pH:8, ETA999; SkyTec Laboratories, UT, USA), and decorin antibody (citrate buffer, pH:6, CBB999; SkyTec Laboratories, UT, USA), and heated in a microwave oven. Hydrogen peroxidase $\left(\mathrm{H}_{2} \mathrm{O}_{2}\right.$; ACA125; SkyTec Laboratories, UT, USA) was used as a blocking agent for $10 \mathrm{~min}$, while AAA125 (SkyTec Laboratories, UT, USA) was used for protein blocking for $5 \mathrm{~min}$. As a VEGF antibody, clon G1 (GeneTex Inc., CA, USA) was used in 1:100 dilution and incubated for one hour. As a decorin antibody, clone polyclonal (GeneTex Inc., CA, USA) was used in 1:500 dilution and incubated for one hour. As secondary antibodies, the SHP125 SensiTek HRP Anti-Polyvalent (SkyTec Laboratories, UT, USA) and ACK125 DAB chromogen (SkyTec Laboratories, UT, USA) were used. In addition, ready-to-use kits (SkyTec Laboratories, UT, USA) were used.

The IHC staining was applied to the endometrium of both patient and control groups and to the ovarian of both patient and control groups. The IHC stains of decorin were evaluated by a single experienced pathologist using a four-point semi-quantitative scale for staining intensity of endometrial stromal and epithelial/glandular cells: 0 (no staining), 1 (weak), 2 (strong), and 3 (very strong) (1). For IHC scoring of VEGF, a four-point semi-quantitative scale for staining intensity of ovarian stromal and epithelial/glandular cells: 0 (no staining), 1 (<20\%), 2 (20 to $60 \%)$, and $3(\geq 60 \%)$. ${ }^{16}$

Descriptive data were expressed in mean \pm standard deviation (SD), median (min-max) for quantitative data and number and percentage for categorical variables, where applicable.The Shapiro-Wilk test was used to test the normal distribution of the quantitative variables. Independent samples $t$-test was used to compare the groups in terms of age and body mass index (BMI). The Mann-Whitney U-test was performed to compare the groups based on the VEGF and decorin staining intensity scores of endometrial and ovarian tissues. The Spearman rank correlation was used to evaluate possible relationship between the IHC staining (VEGF versusdecorin). A p-value of $<0.05$ was considered statistically significant.Statistical analysis was performed using the Jamovi Project version 1.0 free software (www.jamovi.org).

\section{RESULTS}

A total of 30 patients underwent TAH+BSO/USO for endometriomas $(n=20)$ and for other benign pathologies $(n=10)$. 
Table I: Staining intensity scores of endometrial and ovarian tissues.

\begin{tabular}{|c|c|c|c|c|c|c|c|}
\hline & Group & $\mathbf{N}$ & Mean & Median & $25^{\text {th }}-75^{\text {th }}$ & SD & p* \\
\hline \multirow[b]{2}{*}{ VEGF (endometrial) } & Control group & 10 & 1.000 & 1.00 & $1-1$ & 0.471 & \multirow{2}{*}{$<0.001$} \\
\hline & Patient group & 20 & 1.850 & 2.00 & $2-2$ & 0.366 & \\
\hline \multirow[b]{2}{*}{ VEGF (ovarian) } & Control group & 10 & 0.800 & 1.00 & $0.75-1$ & 0.422 & \multirow{2}{*}{$<0.001$} \\
\hline & Patient group & 20 & 1.800 & 2.00 & $1.25-2$ & 0.523 & \\
\hline \multirow[b]{2}{*}{ Decorin (endometrial) } & Control group & 10 & 2.100 & 2.00 & $2-2.25$ & 0.568 & \multirow{2}{*}{$<0.001$} \\
\hline & Patient group & 20 & 0.950 & 1.00 & $1-1$ & 0.394 & \\
\hline \multirow{2}{*}{ Decorin (ovarian) } & Control group & 10 & 2.000 & 2.00 & $2-2$ & 0.471 & \multirow{2}{*}{0.015} \\
\hline & Patient group & 20 & 1.350 & 1.00 & $1-2$ & 0.587 & \\
\hline
\end{tabular}

Table II: Correlation analysis results between staining patterns.

\begin{tabular}{|l|c|c|c|c|c|}
\hline \multicolumn{2}{|c|}{} & \multicolumn{2}{c|}{ Spearman } \\
\cline { 3 - 6 } \multicolumn{2}{|c|}{} & \multicolumn{2}{c|}{$r$} & $p$ \\
\hline VEGF (endometrial) & - & VEGF (ovarian) & 0.571 & $* * *$ & $<0.001$ \\
\hline VEGF (endometrial) & - & $\begin{array}{c}\text { Decorin } \\
\text { (endometrial) }\end{array}$ & -0.600 & $* * *$ & $<0.001$ \\
\hline VEGF (endometrial) & - & Decorin (ovarian) & -0.467 & $* *$ & 0.009 \\
\hline VEGF (ovarian) & - & $\begin{array}{c}\text { Decorin } \\
\text { (endometrial) }\end{array}$ & -0.644 & $* * *$ & $<0.001$ \\
\hline VEGF (ovarian) & - & Decorin (ovarian) & -0.589 & $* * *$ & $<0.001$ \\
\hline Decorin (endometrial) & - & Decorin (ovarian) & 0.453 & $*$ & 0.012 \\
\hline$* p<.05, * * p<.01, * * * p<001$. VEGF: Vascular endothelial growth factor. \\
\hline
\end{tabular}

The mean age and BMIwere $46.7 \pm 25$ years and $27.3 \pm \mathrm{Kg} / \mathrm{m}^{2}$ in the patient group and $49.5 \pm 2.95$ years and $25.7 \pm \mathrm{Kg} / \mathrm{m}^{2}$ in the control group, indicating no statistically significant difference between the groups, $p=0.104$ and $p=0.774$. In addition, there was no significant difference in the gravidity and parity of both groups $p=0.772$ and $p=0.383$ ).

Of the patient group, $11(55 \%)$ had an accompanying uterine myoma and one (5\%) had an endometrial polyp. The control group consisted of 10 patients operated for benign gynecological pathologies, except for adnexal masses and seven (70\%) had a uterine myoma, two (20\%) had prolapse, and one (10\%) had an endometrial polyp.

Table I shows the staining intensity scores of endometrial and ovarian tissues between the groups. Accordingly, VEGF staining revealed a significantly higher intensity of staining in both endometrial and ovarian tissues in patients with endometrioma compared to the control group $(p<0.001$ and $p$ $<0.001$ ). However, decorin staining showed a significantly higher intensity of staining in both endometrial and ovarian tissues in patients operated for other benign pathologies (control group, $p<0.001$ and $p=0.015$ ) (Figure 1 ).

Correlation of the endometrial and ovarian tissues are shown in Table II. Accordingly, there was a statistically significant negative correlation between the VEGF and decorin staining of endometrial tissues (concordance rate $-0.600, p=0.001$ ). Similarly, there was a statistically significant negative correlation between the VEGF and decorin staining of ovarian tissues (Table II, concordance rate $-0.589, \mathrm{p}<0.001$ ).

\section{DISCUSSION}

In the present study, the IHC staining of decorin and VEGF of ovarian and endometrial tissues in patients with and without endometriosis was evaluated and the role of decorin and VEGF in ovarian endometriosis was investigated. The study results showed a lower-intensity staining of decorin, but a higher-intensity staining of VEGF in both ovarian and endometrial tissues in patients with endometriosis compared to the control group.

Previous studies including patients with endometriosis have shown that ectopic endometrial tissues expressed VEGF much more than eutopic endometrial tissues, particularly at the luteal phase of the menstrual cycle., ${ }^{8,17,18}$ In a study comparing patients with endometriosis and healthy controls, Di Carlo et al. collected endometrial biopsy samples and performed IHC staining and real-time polymerase chain reaction (RT-PCR) to analyse the expression of VEGF, metalloproteinase, and angiopoietin 1 and $2 .{ }^{17}$ They observed that the levels of angiogenic factors and metalloproteinases were higher in endometriotic cysts than eutopic endometrium in patients with endometriosis and these substances were overexpressed in eutopic endometrium of patients with endometriosis, compared to healthy controls. There is a growing number of evidence suggesting that eutopic endometrium behaves differently in patients with endometriosis than those without endometriosis. ${ }^{18}$ In the present study, a higher intensity of staining of VEGF of both ectopic and eutopic endometrial tissues in the patients with endometriosis was found. This finding supports the notion that VEGF and angiogenesis play a key role in the formation and maintenance of ectopic endometrial tissues. ${ }^{18}$ Furthermore, a positive and statistically significant correlation between the VEGF staining of both ovarian and endometrial tissues of the patients with endometriosis was found. Similar staining pattern in both tissues indicates that angiogenesis is dense in both of these tissues and endometriosis is a systemic condition. 

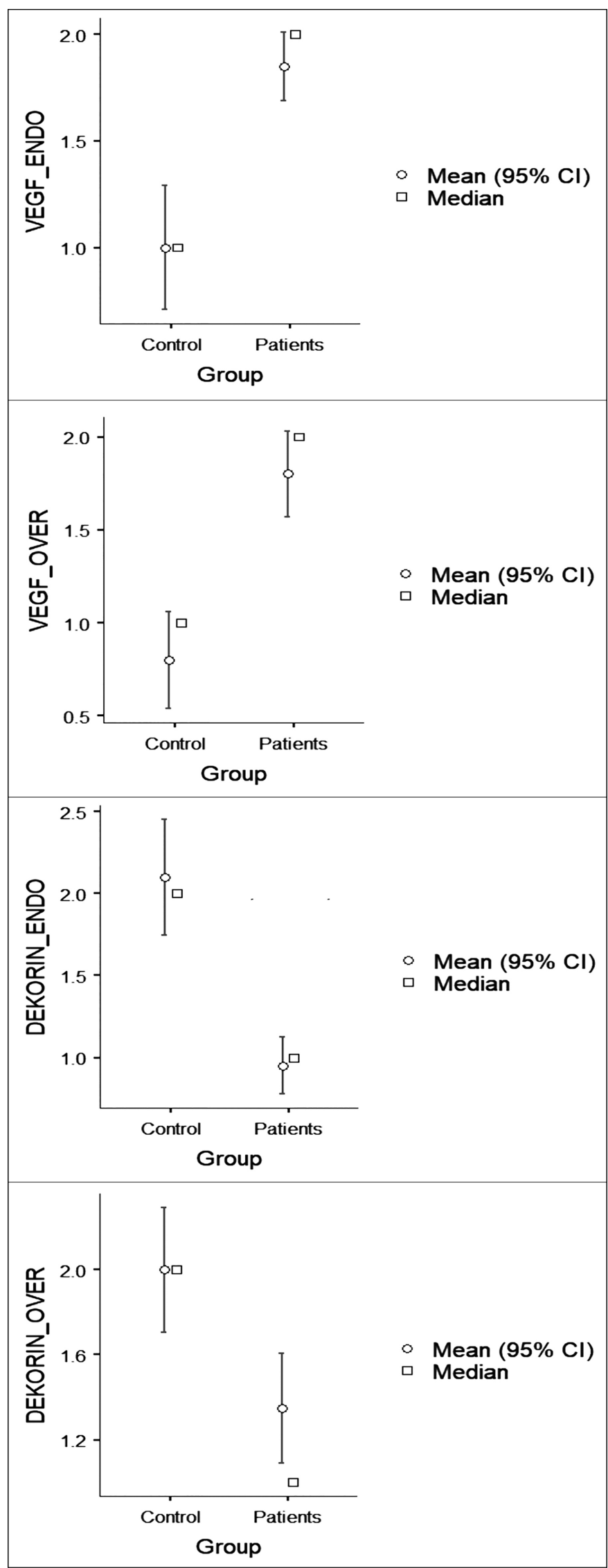

Figure 1: Descriptive staining results.
In recent years, the concept of anti-angiogenic treatment has been extensively discussed in the treatment of endometriosis. Since the VEGF and VEGF receptor (VEGFR) play a major role in angiogenesis, there is a growing number of studies investigating the efficacy of anti-VEGF antibodies and VEGFR inhibitors. ${ }^{19,20}$ In a review and meta-analysis including studies examining the efficacy of anti-VEGF/VEGFR agents in animal models, anti-VEGF/VEGFR treatment was effective in inhibiting the growth of endometriotic lesions and relieving pain. ${ }^{19}$ Rein et al. evaluated a VEGF-targeted gene therapy using conditionally replicative adenovirus for the treatment of endometriosis and performed VEGF IHC staining and gene expression using RT-PCR in eutopic endometrium and endometriotic lesions. ${ }^{19}$ They concluded that VEGF was a promising promoter with sufficient specificity for targeting of endometriotic cells.

It is well established that the interaction between decorin and VEGF induces anti-angiogenic and pro-autophagic effects; and angiogenesis plays a central role in the pathogenesis of endometriosis. In the light of these data, the present study was designed to investigate the relationship between the VEGF and decorin in patients with endometriosis.

Currently, treatment of endometriosis includes surgical removal of the endometriotic foci or pharmacological treatments such as gonadotropin-releasing hormone ( $\mathrm{GnRH}$ ) analogues, aromatase inhibitors, or oral contraceptives which inhibit the endogenous estrogen secretion. ${ }^{2,21}$ Although agents used in medical treatment are helpful for symptomatic relief, recurrence after the discontinuation of treatment and possible side effects limit their use in the long-term. ${ }^{22}$ Therefore, it is of paramount importance to develop novel therapeutic agents in the treatment of endometriosis. Since angiogenesis plays a role in the pathogenesis of endometriosis and decorininhibits angiogenesis through a variety of pathways, it seems to be wise to develop novel therapeutic regimens through decorin in the treatment of endometriosis.

Dienogest is a fourth-generation progestin and is effective in relieving pelvic pain and shrinking endometriotic lesions with a similar rate to $\mathrm{GnRH}$ analogues. ${ }^{23}$ In a study, Ono et al. ${ }^{1}$ evaluated patients aged 20 to 50 years who had a regular menstrual cycle and had a histopathologically confirmed diagnosis of endometriosis. The patients were divided into two groups as those receiving dienogest for three to five months and those not receiving dienogest. Decorin mRNA expression was significantly higher in the patients treated with dienogest. The authors concluded that dienogest increased the p21 expression in the endometriotic epithelial and stromal cells.

Decorinfunctions as a signal molecule, particularly in the regulation of inflammation and autophagy. ${ }^{24}$ It increases the expression of paternally expressed gene 3 (Peg3) in the tumor tissue and evokes autophagy in endothelial cells, slowing the progression. ${ }^{24}$ Some authors have shown that decorin has partial agonistic effects for VEGFR-2 in the 
endothelial cells, resulting in Peg3-dependent autophagy. ${ }^{24}$ In addition, soluble decorin promotes intracellular catabolism of endothelial VEGF-A. ${ }^{13}$ Similarly, Yan et al.reported that adenovirus E1A increased the decorin expression in lung cancer cells and direct interaction between decorin and VEGF increased the proteasomal degradation of VEGF. ${ }^{25}$ Thus, the interaction between adenovirus E1A and decorin induced alterations in the vasculogenesis and angiogenesis, resulting in the regulation of the ECM. In the current study, a lower-intensity staining of decorin in both ovarian and endometrial tissues was observed in patients with endometriosis compared to the control group. In addition, there was a negative and statistically significant correlation between the VEGF and decorin staining of ovarian and endometrial tissues, consistent with the existing literature.

Nonetheless, there are some limitations to this study. First, this study has a retrospective design with a relatively small sample size. Second, this study was designed to investigate the relationship between the VEGF and decorin in patients with and without endometriosis. However, the mRNA expression of the VEGF and decorinwas unable to be examined due to retrospective nature of the study. Third, the IHC staining results were interpreted by a single pathologist and no intraobserver reliability was tested. On the other hand, the main strength of the present study is that this is the first study to examine the relationship between decorin and VEGF using IHC technique in patients with endometriosis. The authors believe that these results would provide a contribution to the body of knowledge on this topic and shed light into the therapeutic implications of decorin in the treatment of endometriosis in the future. However, further large-scale, prospective studies evaluating tissue measurements are warranted to confirm these findings.

\section{CONCLUSION}

This study results showed significant differences between the IHC staining of both endometrial and ovarian tissues using VEGF and decorin in patients with endometriosis and those operated for other benign pathologies. There was also a negative, but significant correlation between the two staining patterns. Angiogenesis plays a critical role in endometriosis, as in all tumoral tissues, and the interaction between decorin and VEGF suggests that decorin may be a promising molecule for the treatment of endometriosis.

\section{FUNDING:}

This study was funded by the University of Health Sciences Project No. BAP-2019/096.

\section{ETHICAL APPROVAL:}

The protocol for the current study has been approved by the Ethics Committee of Zeynep Kamil Women's and Children's Disease Training and Research Hospital (Approval No. 6.3.2019 32).

\section{PATIENTS' CONSENT:}

A written informed consent was obtained from each patient.

\section{CONFLICT OF INTEREST:}

The authors declared no conflict of interest.

\section{AUTHORS' CONTRIBUTION:}

GAA, HA, NK, NT, OD: Designed the study, searched the literature, planned the concept, performed statistical analysis, and prepared and edited the manuscript.

All authors read and approved the final manuscript.

\section{REFERENCES}

1. Ono YJ, Terai Y, Tanabe A, Hayashi A, Hayashi M, Yamashita $Y$, et al. Decorin induced by progesterone plays a crucial role in suppressing endometriosis. J Endocrinol 2014; 223(2): 203-16. doi: 10.1530/JOE-14-0393.

2. Vercellini P, Viqanò $P$, Somiqliana E, Fedele L. Endometriosis: Pathogenesis and treatment. Nat Rev Endocrinol 2014; 10(5):261-75.doi: 10.1038/nrendo.2013. 255.

3. Lucidi RS, Witz CA, Chrisco M, Binkley PA, Shain SA, SchenkenRS.A novel in vitro model of the early endometriotic lesion demonstrates that attachment of endometrial cells to mesothelial cells is dependent on the source of endometrial cells. Fertil Steril 2005; 84(1): 16-21. doi: 10. 1016/j.fertnstert.2004.10.058.

4. Bulun SE, Yilmaz BD, Sison C, Miyazaki K, Bernardi L, Liu S, et al. Endometriosis. Endocr Rev 2019; 40(4):1048-1079. doi: 10.1016/j.fertnstert.2004.10.058

5. Kim JJ, Kurita T, Bulun SE. Progesterone action in endometrial cancer, endometriosis, uterine fibroids, and breast cancer. Endocr Rev 2013; 34(1):130-62. doi: 10.1210/ er.2012-1043.

6. Barragan F, Irwin JC, Balayan S, Erikson DW, Chen JC, Houshdaran $\mathrm{S}$, et al. Human endometrial fibroblasts derived from mesenchymal progenitors inherit progesterone resistance and acquire an inflammatory phenotype in the endometrial niche in endometriosis. Biol Reprod 2016; 94(5):118.doi: 10.1095/biolreprod.115.136010.

7. Attia GR, Zeitoun K, Edwards D, Johns A, Carr BR, Bulun SE. Progesterone receptor isoform $A$ but not $B$ is expressed in endometriosis. J Clin Endocrinol Metab 2000; 85(8): 2897-2902.Progesterone receptor isoform $A$ but not $B$ is expressed in endometriosis.

8. Donnez J, Smoes P, Gillerot S, Casanas-Roux F, Nisolle M. Vascular endothelial growth factor (VEGF) in endometriosis. Hum Reprod 1998; 13(6):1686-90.

9. McLaren J. Vascular endothelial growth factor and endometriotic angiogenesis. Hum Reprod Update 2000; 6(1):45-55.doi: 10.1093/humupd/6.1.45.

10. Neve A, Cantatore FP, Maruotti N, Corrado A, Ribatti D. Extracellular matrix modulates angiogenesis in physiological and pathological conditions. BioMed Res Int2014; 2014: 756078. doi: 10.1155/2014/756078.

11. Iozzo RV. The family of the small leucine-rich proteoglycans: Key regulators of matrix assembly and cellular growth. Crit Rev Biochem Mol Biol 1997; 32(2): 141-74.doi: 10.3109/ 
10409239709108551.

12. Neill T, Painter H, Buraschi S, Owens RT, Lisanti MP, Schaefer $L$, et al. Decorin antagonizes the angiogenic network: concurrent inhibition of Met, hypoxia inducible factor lalpha, vascular endothelial growth factor $A$, and induction of thrombospondin-1 and TIMP3. J Biol Chem 2012; 287(8):5492-506.doi: 10.1074/jbc.M111. 283499.

13. Neill T, Chen CG, Buraschi S, Iozzo RV. Catabolic degradation of endothelial VEGFA via autophagy. J BiolChem 2020; 295(18):6064-6079. doi: 10.1074/jbc. RA120.012593.

14. Carmeliet P. Angiogenesis in health and disease. Nat Med 2003; 9:653-60

15. Muzii L, Bianchi A, Bellati F, Cristi E, Pernice M, AZullo M, et al. Histological analysis of endometriomas: What the surgeon needs to know. FertilSteril 2007; 87(2):362-6.doi: 10.1016/j.fertnstert.2006.06.055.

16. Rein DT, Schmidt T, Bauerschmitz G, Hampl M, Beyer IM, Paupoo AA, et al.Treatment of endometriosis with a VEGF-targeted conditionally replicative adenovirus. Fertil Steril 2010; 93(8):2687-94. doi: 10.1016/j. fertnstert. 2009.04.042.

17. Di Carlo C, Bonifacio M, Tommaselli GA, Bifulco G, Guerra G, Nappi C. Metalloproteinases, vascular endothelial growth factor, and angiopoietin 1 and 2 in eutopic and ectopic endometrium. FertilSteril 2009;91(6):2315-23.doi: 10. 1016/j. fertnstert.2008.03.079.

18. Bourlev V, Volkov N, Pavlovitch S, Lets N, Larsson A, OlovssonM.The relationship between microvessel density, proliferative activity and expression of vascular endothelial growth factor-A and its receptors in eutopic endometrium and endometriotic lesions. Reproduction 2006; 132(3): 501-9. doi: 10.1530/rep.1.01110.

19. Liu S, Xin X, Hua T, Shi R, Chi S, Jin Z, et al.Efficacy of antiVEGF/VEGFR agents on animal models of endometriosis: $A$ systematic review and meta-analysis. PLOS One 2016; 11(11): e0166658. doi: 10.1371/journal.pone. 0166 658.

20. Song WW, Lu H, Hou WJ, Xu GX, Zhang JH, Sheng YH, et al.Expression of vascular endothelial growth factor $\mathrm{C}$ and anti-angiogenesis therapy in endometriosis. Int J ClinExpPathol 2014;7(11):7752-59.

21. Vercellini P, Eskenazi B, Consonni D, Somigliana E, Parazzini F, Abbiati A, et al. Oral contraceptives and risk of endometriosis: A systematic review and metaanalysis. HumReprod Update 2011;17(2):159-170.doi: 10.1093/humupd/dmq042.

22. Brown J, Pan A, Hart RJ.Gonadotrophin-releasing hormone analogues for pain associated with endometriosis. Cochrane Database Sys Rev 2010; 2010(12): CD00 8475.doi: 10. 1002/14651858.CD008475.

23. Felice P, Daniela H, Christian S, Thomas F, Christoph G, Stefano L,et al. Reduced pelvic pain in women with endometriosis: Efficacy of lonq-term dienogest treat ment. Archives Gynecol Obstetrics 2012; 285(1):167-73. doi: 10.1007/s00404-011-1941-7.

24. Buraschi S, Neill T, Goyal A, Poluzzi C, Smythies J, Owens $\mathrm{RT}$, et al. Decorin causes autophagy in endothelial cells via Peg3. Proc Natl Acad Sci U S A 2013;110(28):E2582-E2591. doi: 10.1073/pnas.1305732110.

25. Ge Y, Zhang W, Qin J, Zhang C, Tian W, Zhang Q, et al.A novel role mediated by adenoviral E1A in suppressing cancer through modulating decorin. Med Oncol 2019; 36(12): 96. doi: 10.1007/s12032-019-1325-6. 